\title{
Uso e eficácia clínica do laser no tratamento da hipersensibilidade dentinária: uma revisão de literatura
}

Use and clinical efficacy of laser in the treatment of dentin hypersensitivity: a literature review Uso y eficacia clínica del láser en el tratamiento de la hipersensibilidad dentinaria: una revisión de la literatura

Erika Thaís Cruz SILVA ${ }^{\mathbf{1}}$

Rodrigo Gadelha VASCONCELOS ${ }^{2}$

Marcelo Gadelha VASCONCELOS ${ }^{2}$

${ }^{I}$ Graduada em Odontologia, Universidade Estadual da Paraíba (UEPB) Araruna - PB, Pós-Graduanda em Cirurgia Oral Menor, COESP, João- Pessoa-PB, Brasil ${ }^{2}$ Professor Doutor do Departamento de Odontologia da Universidade Estadual da Paraíba - UEPB, 58429-500 Araruna - PB, Brasil

\section{Resumo}

Introdução: A hipersensibilidade dentinária representa uma dor aguda e de curta duração em resposta à um estímulo. Ao longo do tempo, vários tratamentos para a hipersensibilidade dentinária têm surgido, entre eles, destaca-se o uso do laser. Objetivo: Realizar uma revisão da literatura acerca dos estudos que abordem o uso dos lasers sejam eles de alta ou de baixa potência, destacando sua eficácia clínica no tratamento da hipersensibilidade dentinária. Materiais e método: Realizou-se uma revisão bibliográfica de estudos disponíveis na literatura publicados nos últimos 10 anos (2008- 2018), por meio da busca bibliográfica nas bases de dados eletrônicos utilizando os seguintes descritores: Lasers, hipersensibilidade dentinária e dor dentinária. Resultados: Podem ser utilizados tanto o laser de alta quanto o laser de baixa potência, de forma que cada um atua por um mecanismo diferente no tratamento da hipersensibilidade dentinária. Conclusão: Apesar de existir uma grande variabilidade nos protocolos e dosagens utilizados, de uma forma geral, os lasers mostram-se eficazes no tratamento da hipersensibilidade dentinária.

Descritores: Lasers; Sensibilidade da Dentina; Terapêutica.

\section{Abstract}

Introduction: Density hypersensitivity represents acute and short-lived pain in response to a stimulus. Over time, several treatments for dentin hypersensitivity have arisen, among them, the use of laser is prominent. Objective: To carry out a review of the literature on the studies that address the use of lasers be they high or low power, highlighting its clinical effectiveness in the treatment of dentin hypersensitivity. Materials and methods: A literature review was carried out in the literature published in the last 10 years (2008-2018), through a bibliographic search in the electronic databases using the following descriptors: Lasers, dentin hypersensitivity and dentin pain. Results: Both high and low power laser can be used, so that each one acts by a different mechanism in the treatment of dentin hypersensitivity. Conclusion: Although there is great variability in the protocols and dosages used, lasers are generally effective in the treatment of dentin hypersensitivity.

Descriptors: Lasers; Dentin Sensitivity; Therapeutics.

\section{Resumen}

Introducción: La hipersensibilidad de la dentina representa un dolor agudo y de corta duración en respuesta a un estímulo. A lo largo del tiempo, varios tratamientos para la hipersensibilidad de la dentina han surgido, entre ellos, se destaca el uso del láser. Objetivo: Realizar una revisión de la literatura acerca de los estudios que aborden el uso de los láseres sean de alta o de baja potencia, destacando su eficacia clínica en el tratamiento de la hipersensibilidad dentinaria. Materiales y método: Se realizó una revisión bibliográfica de estudios disponibles en la literatura publicados en los últimos 10 años (2008- 2018), por medio de la búsqueda bibliográfica en las bases de datos electrónicos utilizando los siguientes descriptores: Láser, hipersensibilidad de la dentina y dolor de la dentina. Resultados: Se pueden utilizar tanto el láser de alta y el láser de baja potencia, de forma que cada uno actúa por un mecanismo diferente en el tratamiento de la hipersensibilidad de la dentina. Conclusión: A pesar de que existe una gran variabilidad en los protocolos y dosificaciones utilizadas, en general, los láseres se muestran eficaces en el tratamiento de la hipersensibilidad de la dentina.

Descriptores: Rayos Laser; Sensibilidad de la Dentina; Terapéutica.

INTRODUÇÃO

A hipersensibilidade dentinária representa uma dor aguda e de curta duração que acontece em resposta à um estímulo. No entanto, com a remoção do estímulo a dor tende a desaparecer. Dentre os estímulos que podem desencadear a hipersensibilidade dentinária, exemplificam-se os térmicos, táteis, osmósticos ou químicos ${ }^{1}$.

Em condições normais, a dentina não é capaz de sofrer estímulos dolorosos pois é protegida pelo esmalte ou pelo cemento; contudo, com a exposição das terminações periféricas dos túbulos dentinários uma forte sensibilidade poderá ser desencadeada ${ }^{2}$.

A hipersensibilidade acontece geralmente quando os túbulos dentinários são expostos ao meio bucal por meio das lesões cervicais não cariosas (erosão, abrasão, abfração, atrição), dentes fraturados com dentina exposta ou por tratamentos periodontais. Essas lesões afetam com maior frequência os caninos e pré-molares sendo mais prevalentes na faixa etária de 30 à 40 anos. A superfície vestibular é a região dos dentes mais acometida ${ }^{3,4}$.

Existem diversas teorias que buscam explicar como acontece o mecanismo da hipersensibilidade dentinária, porém, a mais aceita atualmente é a teoria hidrodinâmica. Segundo essa teoria, os estímulos que entram em contato com a superfície dentinária exposta são capazes de causar um movimento de fluido dentro dos túbulos dentinários. Em decorrência disso, as fibras nervosas presentes no complexo dentino-pulpar serão estimuladas causando, assim, uma sensibilidade dolorosa ${ }^{5}$.

Ao longo do tempo, vários tratamentos para a hipersensibilidade dentinária têm surgido. Eles vão desde à utilização de dentifrícios específicos, flúor, agentes dessensibilizantes, adesivos dentinários, restaurações e também os lasers. Contudo, apesar da grande disponibilidade de formas de tratamento para a hipersensibilidade dentinária, o principal desafio parece consistir em encontrar uma substância que seja eficaz em um curto espaço de tempo, que não cause recidiva da hipersensibilidade e que elimine efetivamente a sensação dolorosa ${ }^{5}$.

A determinação do tratamento mais adequado e efetivo para tratar a hipersensibilidade dentinária têm 
tido uma constante busca; vários estudos têm sido realizados com o objetivo de verificar a eficácia terapêutica das diversas modalidades de tratamento para esta condição. Segundo Asnaashari e Moeni ${ }^{6}$ um tratamento ideal para a hipersensibilidade dentinária deve agir rápido, ser eficaz por longos períodos, ou seja, duradouro, ser de fácil aplicação, não irritar a polpa, não causar dor e não manchar os dentes. Os dois principais métodos de tratamento da hipersensibilidade dentinária acontecem através da oclusão dos túbulos dentinários e também pelo bloqueio da atividade nervosa.

Os lasers podem ser classificados em lasers de alta potência ou alta intensidade e de baixa potência ou baixa intensidade; apresentam algumas características que diferem a luz produzida pelo laser da luz comum, são elas: apresenta comprimento de onda único, unidirecional, formada em uma fonte monocromática, coerente e possui a propriedade de transportar altas concentrações de energia em seu feixe ${ }^{7}$.

De forma geral, os lasers de baixa potência são caracterizados por promoverem ação cicatrizadora e efeitos de bioestimulação nos tecidos. Eles são bem tolerados pelos pacientes e podem ser utilizados em diversas situações clínicas, seja de forma isolada ou como coadjuvante à outros tratamentos. Já os lasers de alta potência possuem efeitos de coagulação, cauterização e vaporização dos tecidos ${ }^{7}$.

Para o tratamento da hipersensibilidade dentinária podem ser usados tantos os lasers de alta potência quanto os lasers de baixa potência. Entretanto, seus mecanismos de ação são diferentes, de forma que, os lasers de alta potência vão atuar promovendo obliteração dos túbulos dentinários. Por outro lado, os lasers de baixa potência possuem sua ação a nível celular sendo capazes de promoverem efeitos analgésicos, anti-inflamatório e bioestimulador da polpa dental levando a formação de dentina reacional ${ }^{8}$.

A dosagem de aplicação do laser de baixa intensidade deve ser em torno de 3,5 a $4,0 \mathrm{~J} / \mathrm{cm}$. Já para os lasers de alta intensidade é recomendável que a irradiação seja realizada com potências inferiores a $1,5 \mathrm{~W}$ estando o valor ideal entre 0,25 a $0,75 \mathrm{~W}^{9}$.

Ao irradiar um tecido duro dentário com o laser, alterações morfológicas e químicas serão provocadas. Todavia, são as características de absorção do tecido que determinarão a extensão dessas alterações. Dessa forma, o tipo de laser e tecido dental irradiado afetarão a forma como as mudanças ocorrem ${ }^{10}$.

Segundo Ozlem et al. ${ }^{11}$ os parâmetros do laser que afetam a quantidade de energia aplicada a uma determinada superfície incluem nível de potência (W), tempo de exposição (segundos), densidade de energia $\left(\mathrm{J} / \mathrm{cm}^{2}\right)$, distância da superfície e o ângulo entre o tecido alvo e a ponta do laser.

A terapia a laser no tratamento da hipersensibilidade dentinária consiste em uma alternativa que vem sendo aplicado na prática clínica com resultados promissores. Diante do exposto, o presente trabalho tem como objetivo realizar uma revisão da literatura acerca dos estudos que abordem este assunto, destacando a eficácia clínica dos lasers, sejam eles de alta ou de baixa potência, que são aplicados no tratamento da hipersensibilidade dentinária.

\section{MATERIAL E MÉTODO}

Realizou-se uma revisão bibliográfica de estudos disponíveis na literatura publicados nos últimos 10 anos (2008- 2018), por meio da busca bibliográfica nas bases de dados eletrônicos PubMED, Lilacs, Science Direct, Scielo (Scientific Eletronic Library) e Google Acadêmico. Para a pesquisa foram utilizados os seguintes descritores: Lasers, hipersensibilidade dentinária e dor dentinária.

Como critérios de inclusão, foram adotados os artigos escritos em inglês, espanhol e português; aqueles que se enquadravam no enfoque e objetivo do trabalho e os mais relevantes em termos de delineamento das informações desejadas. Assim como, foi indispensável à disponibilidade do texto integral para sua inclusão no estudo.

Foram excluídos da amostra os artigos que não apresentaram relevância sobre o tema abordado, bem como trabalhos de conclusão de curso, teses, dissertações e aqueles estudos que não se enquadraram nos critérios de inclusão.

RESULTADOS E DISCUSSÃO

As evidências do potencial de tratamento da hipersensibilidade dentinária com o uso de lasers surgiram em 1985. A partir de então, muitos estudos passaram a ser realizados com o objetivo de obter diversas informações acerca da aplicação desses lasers no tratamento da hipersensibilidade dentinária ${ }^{6}$.

O efeito de dessensibilização depende do tipo de laserterapia utilizado, sendo assim, acredita-se que diferentes tipos de lasers parecem possuir mecanismos diferentes. Os lasers utilizados no tratamento da hipersensibilidade dentinária podem ser divididos em dois grupos: 1- Lasers de baixa potência (baixa intensidade), que são representados pelos hélio-neônio (He-Ne) e lasers de gálioaluminossenido de sódio (GaAlAs) (diodos) e 2Lasers de alta potência (alta intensidade) representados pelos laser de dióxido de carbono $\left(\mathrm{CO}_{2}\right)$, granada de ítrio-alumínio dopado com neodímio ou de érbio (lasers Nd: YAG, Er: YAG) e érbio, cromo dopado: ítrio, escândio, gálio e granada (Er, Cr: YSGG) ${ }^{6}$.

Sabendo que os mecanismos de ação dos lasers de alta e baixa intensidade na redução da 
hipersensibilidade dentinária são diferentes, Tabibzadeh et al. ${ }^{12}$ realizaram um estudo que verificou o efeito dessensibilizante da aplicação combinada de lasers com duas saídas diferentes de potências (alta e baixa intensidade) e comparou-a com a terapia única do laser de alta intensidade. Os resultados mostraram que não houve diferença estatisticamente significante entre as duas formas de tratamento utilizadas.

Para que a hipersensibilidade dentinária seja tratada com sucesso é importante que o tratamento consiga resistir aos desafios ácidos e mecânicos presentes na cavidade oral. Sabe-se que os agentes dessensibilizantes quando expostos a alimentos ácidos ou escovação dentária podem ser removidos da superfície dentária e, portanto, não possuem um efeito duradouro. Nesse sentido, a utilização dos lasers para o tratamento da hipersensibilidade dentinária representa uma alternativa eficaz, tendo em vista que possuem um efeito a longo prazo e baixa solubilidade em ácido ${ }^{13}$.

Contudo, segundo Lopes et al. $^{14}$ ainda existem dúvidas quanto à aplicação dos lasers no tratamento da hipersensibilidade dentinária, principalmente no que concerne ao correto protocolo, quanto ao uso de altas ou baixas dosagens e até mesmo na associação dos lasers com dessensibilizantes.

\section{- Lasers de alta intensidade}

Os lasers de alta intensidade atuam obliterando a embocadura dos túbulos dentinários; isso acontece por meio da irradiação direta do tecido que está exposto. Em decorrência da irradiação, serão formadas alterações morfológicas na dentina com consequente selamento dos túbulos dentinários com até $4 \mu \mathrm{m}$ de profundidade ${ }^{9}$.

A efetividade dos lasers de alta intensidade no tratamento da hipersensibilidade dentinária acontece devido à fusão que ocorre no tecido dentinário como resultado da transmissão de calor proporcionada pelo laser. Essa interação resulta em uma fusão e solidificação da dentina com consequente selamento e redução no diâmetro dos túbulos dentinários ${ }^{15}$.

Dentre esses lasers, alguns exemplos que podem ser utilizados para o tratamento da hipersensibilidade dentinária são os neodímio: ítrioalumínio-granada (Nd:YAG), érbio: ítrio-alumíniogranada (Er:YAG) e érbio: ítrio-escândio-gáliogranada (Er, Cr:YSGG) e dióxido de carbono $\left(\mathrm{CO}_{2}\right)^{9}$.

Belal e Yassin ${ }^{10}$ realizaram um estudo para avaliar e comparar os efeitos do uso de laser $\mathrm{CO}_{2}$ ou o laser Er:YAG no tratamento da hipersensibilidade dentinária. Neste estudo, eles puderam concluir que tanto o laser $\mathrm{CO}_{2}$ quanto o Er:YAG são capazes de reduzir significativamente os sintomas da hipersensibilidade dentinária. Contudo, o laser Er:YAG tem um efeito maior na oclusão dos dentinários com menor alteração térmica. Além do mais, nem o laser de $\mathrm{CO}_{2}$ nem o de Er:YAG têm um efeito significativo sobre a estrutura composicional da superfície afetada, especificamente, sobre o conteúdo mineral.

Segundo Lopes e Aranha ${ }^{16}$ o laser de alta potência que se destaca na literatura e na clínica, nos casos de tratamento para a redução da dor na hipersensibilidade dentinária é o laser Nd:YAG. Além de que, vários estudos comparando a eficácia dos lasers Nd:YAG com outros tipos de tratamento, puderam comprovar sua superioridade na redução da dor e oclusão dos túbulos dentinários. No entanto, mesmo após a irradiação do laser $\mathrm{Nd}$ :YAG não se pode ter certeza que todos os túbulos dentinários foram ocluídos, isso explica o motivo pelo qual alguns pacientes ainda relatam dor mesmo após a irradiação. Este estudo mostrou que o único grupo em que toda a amostra não apresentou dor ao final da avaliação foi o grupo em que o laser $\mathrm{Nd}$ : YAG esteve associado ao agente dessensibilizante Gluma ${ }^{\circledR}$.

Em contrapartida, Ozlem et al. ${ }^{11}$ avaliaram a eficácia das aplicações do agente Gluma ${ }^{\circledR}, \mathrm{Nd}$ : YAG e / ou Er, Cr: YSGG e a combinação deles no tratamento da hipersensibilidade dentinária por um período de 180 dias. Neste estudo eles concluíram que houve uma redução significativa no nível de sensibilidade dentinária após todos os tratamentos. Contudo, apesar do Gluma ${ }^{\circledR}$ e Nd: YAG parecerem ter efeitos semelhantes no tratamento da hipersensibilidade dentinária, foi possível observar que o laser Er, Cr: YSGG com ou sem aplicação de Gluma ${ }^{\circledR}$ foi a modalidade mais efetiva no tratamento da hipersensibilidade dentinária.

Aranha e Eduardo ${ }^{17}$ avaliaram os efeitos dos lasers Er, Cr: YSGG e Er:YAG no tratamento da hipersensibilidade dentinária. Foram incluídos neste estudo 28 pacientes que obedeceram aos critérios de inclusão. Uma escala visual analógica (EVA) foi usada para quantificar a sensibilidade antes, imediatamente depois, 1 semana e 1 mês após o tratamento. Foi possível concluir que tanto os lasers Er,Cr:YSGG quanto o Er:YAG foram adequados para tratamento da hipersensibilidade dentinária e ambos reduziram o nível de dor em 1 mês de acompanhamento. Além disso, todos os dentes mantiveram a vitalidade pulpar com o tratamento. Observou-se, ainda, que o grupo irradiado com o laser Er, Cr:YSGG demonstrou melhores performances na avaliação clínica com uma diminuição mais pronunciada da dor imediatamente após o tratamento.

Os estudos de $\mathrm{Yu}$ e $\mathrm{Chang}^{18}$ tiveram como objetivo avaliar a eficácia clínica do laser Er:YAG na hipersensibilidade dentinária cervical. Para inclusão no estudo, seguindo os critérios de inclusão e exclusão, foram selecionados 20 pacientes. Com o auxílio de uma escala visual analógica (EVA), foi 
solicitado aos participantes que identificassem o grau de sensibilidade térmica que os dentes apresentavam quando estimulados usando um jato de ar de 1 segundo a $1 \mathrm{~cm}$ do dente. O laser Er: YAG foi aplicado a um nível de energia de $60 \mathrm{~mJ} /$ pulso, e uma taxa de repetição de $2 \mathrm{~Hz}$ com irrigação à água. Todos os dentes com hipersensibilidade dentinária foram tratados uma vez e avaliados após quatro semanas. Os resultados evidenciaram que dezoito dos 20 participantes apresentaram redução significativa da hipersensibilidade dentinária em quatro semanas após receber o laser Er: YAG como tratamento.

Ribeiro et al. ${ }^{19}$ avaliaram a influência da escovação e ingestão de bebidas ácidas na longevidade dos tratamentos para hiprrsensibilidade dentinária com o laser de Nd:YAG e agentes dessensibilizantes contendo cloreto de estrôncio e nitrato de potássio. Foi possível concluir que tanto a alimentação quanto a escovação possuem influência na longevidade dos tratamentos para hipersenbilidade dentinária, no entanto, a irradiação com o laser mostrou-se menos vulnerável à dissolução ácida.

Em decorrência dos efeitos térmicos e adversos que podem ser causados na polpa, a energia do laser deve ser aplicada cuidadosamente sobre a superfície dentinária ${ }^{20}$. Namour et al. $^{21}$ realizaram estudo in vitro com o objetivo de avaliar a eficácia de selamento dos túbulos dentinários com o laser $\mathrm{Nd}$ : YAP em diferentes parâmetros e concluiu que esse laser foi capaz de selar os túbulos dentinários sem danificar as superfícies dentinárias e sem prejudicar a vitalidade da polpa. Sendo assim, esse laser pode ser usado com segurança para futuros tratamentos in vivo de hipersensibilidade dentinária, porém em determinadas condições de aplicação.

\section{- Lasers de baixa intensidade:}

Ao contrário do que é observado em outros tipos de tratamento em que o efeito terapêutico principal acontece nos túbulos dentinários expostos, o efeito dos lasers de baixa intensidade no tratamento da hipersensibilidade dentinária, baseia-se na indução de alterações na rede de transmissão nervosa na polpa dentária. Esses lasers induzem a ocorrência de mudanças no potencial elétrico da membrana celular trazendo efeitos anti-inflamatórios, analgésicos e de biomodulação para as células. Além do mais, esta terapia pode, também, estimular a produção de dentina esclerótica, promovendo assim a obliteração interna dos túbulos dentinários ${ }^{9,15}$.

No protocolo de aplicação destes lasers são escolhidos quatro pontos diferentes para aplicação sendo um ponto no ápice da raiz e três pontos na região cervical do dente que apresenta dentina exposta (mésio-vestibular, vestibular e distovestibular) ${ }^{9}$. Os lasers de baixa intensidade mais utilizados para tratamento da hipersensibilidade dentinária são os Hélio-Neônio (He- $\mathrm{Ne}$ ) e Arseneto de Gálio e Alumínio (As-GaAl) 5 .
O comprimento de onda do laser de HélioNeônio (He-Ne) encontra-se na faixa do visível com $632,8 \eta m$ e de coloração vermelha. Já o Arseneto de Gálio e Alumínio (AsGaAl) apresenta comprimento de onda variando entre 620 a $830 \eta \mathrm{m}$, ocupando uma faixa desde $\mathrm{o}$ vermelho até próximo do infravermelho ${ }^{22}$.

Em um estudo realizado por Bal et al. ${ }^{23}$ buscou-se comparar a eficácia do laser de baixa potência e dessensibilizantes contendo $8 \%$ de arginina-carbonato de cálcio no tratamento de dentes com hipersensibilidade dentinária e, também, avaliar se seu uso combinado melhoraria a eficácia do tratamento através de um efeito aditivo ou sinérgico. Foram incluídos no estudo 21 pacientes com 156 dentes afetados. Os dentes foram irradiados pelo tratamento com laser diodo de $685 \mathrm{~nm}$ de comprimento de onda, $25 \mathrm{~mW}, 9 \mathrm{~Hz}$ por $100 \mathrm{seg}$ a 1 $\mathrm{cm}^{2}$ de área $\left(2 \mathrm{~J} / \mathrm{cm}^{2}\right)$ em modo interrompido. Nesse estudo, concluiu-se que a aplicação do laser de baixa potência ou dessensibilizante contendo $8 \%$ de arginina-carbonato de cálcio parece ser eficaz na diminuição da hipersensibilidade dentinária. Entretanto, não houve efeitos aditivos ou sinérgicos na redução da sensibilidade em ambos os tratamentos com combinações.

Dantas et al. ${ }^{22}$ compararam a eficácia do verniz fluoretado (Fluorniz ${ }^{\circledR}$ ) e a irradiação com laser diodo de gálio-arseneto-alumínio (As-GaAl) no tratamento da hipersensibilidade dentinária. Oitenta e seis dentes foram divididos em dois grupos: Grupo 1, dentes tratados com Fluorniz $®$; Grupo 2, dentes irradiados com laser de GaAlAs a $4 \mathrm{~J} / \mathrm{cm}^{2}$ dose. Os dois tratamentos foram aplicados na região cervical vestibular em quatro sessões, com intervalos de 72 à 96 horas. Com os resultados, foi possível concluir que tanto o laser quanto o verniz fluoretado foi eficaz na redução da hipersensibilidade em resposta à estimulação tátil e térmica e evaporativa, contudo, o tratamento com Fluorniz ${ }^{\circledR}$ mostrou-se mais eficaz do que a radiação laser de baixo nível na redução à curto prazo da hipersensibilidade dentinária.

Diversos estudos têm mostrado um melhor efeito terapêutico na combinação entre o laser e os agentes dessensibilizantes no tratamento da hipersensibilidade dentinária. Isso pode ser visto no estudo realizado por Suri et al. ${ }^{24}$ que teve como objetivo comparar a eficácia de $5 \%$ de verniz tópico de $\mathrm{NaF}$ (fluoreto de sódio) e $980 \mathrm{~nm}$ do laser diodo arsenieto de gálio e alumínio ( $\mathrm{GaAlAs}$ ) isolado e, também, a combinação de $5 \%$ de $\mathrm{NaF}+980 \mathrm{~nm}$ laser diodo GaAlAs no manejo da hipersensibilidade dentinária. $\mathrm{O}$ estudo concluiu que o uso combinado de $5 \%$ de verniz de $\mathrm{NaF}$ e $980 \mathrm{~nm}$ de laser diodo GaAlAs resultou em significativa redução na severidade da hipersensibilidade dentinária. $O$ efeito terapêutico dessa combinação é melhor do que a aplicação isolada do laser ou do verniz fluoretado. 
Femiano et al. $^{25}$ realizaram um estudo para comparar a mudança da hipersensibilidade dentinária proveniente de lesões cervicais não cariosas (LCNCs) que não responderam ao tratamento com agentes dessensibilizantes e foram tratadas com restauração de resina composta associada ou não ao uso anterior do laser diodo de baixa potência. Foram incluídos neste estudo 88 dentes com hipersensibilidade proveniente de LCNCs. Os pacientes foram questionados sobre a sensibilidade que apresentavam usando a escala visual analógica (EVA), imediatamente depois, 6 meses e 12 meses após a realização da restauração. Com os resultados, foi possível concluir que a irradiação com o laser diodo realizada anteriormente à restauração promoveu um melhoramento na redução da sintomatologia dolorosa da hipersensibilidade dentinária proveniente de LCNCs tanto imediatamente como em longo prazo.

Lopes et al. ${ }^{14}$ realizaram um estudo que teve como objetivo avaliar diferentes protocolos para tratamento da hipersensibilidade dentinária. Foram utilizados o laser de baixa potência em diferentes dosagens, agentes dessensibilizantes e a associação deles por um período de 6 meses. Neste estudo, foram incluídos 26 pacientes e 55 lesões foram registradas. As lesões foram divididas em cinco grupos de tratamento e avaliação, sendo eles: G1 (Dessensibilizante Gluma ${ }^{\circledR}$ ), G2 (Laser de baixa potência em baixa dose), G3 (Laser de baixa potência em alta dose), G4 (Laser de baixa potência em baixa dose + Dessenbilizante Gluma ${ }^{\circledR}$ ) e G5 (Laser de baixa potência em alta dose + Dessensibilizante Gluma $\AA_{\text {). }}$ O nível de sensibilidade de cada voluntário foi avaliado com uma escala visual analógica (EVA) com uso do ar da seringa tríplice e exploração com a sonda, 5 minutos, 1 semana, 1,3 e 6 meses depois do tratamento. Com os resultados, foi possível concluir que houve efeitos distintos com o uso de diferentes doses do laser de baixa potência, contudo, ambos foram eficientes na redução da dor em 6 meses de acompanhamento clínico. Além do mais, todos protocolos de tratamento foram efetivos na redução da hipersensibilidade dentinária, porém, com diferentes efeitos.

Ko et al. ${ }^{26}$ buscaram avaliar a laserterapia de baixa intensidade no tratamento da hipersensibilidade dentinária por meio do uso de escova dental emissora de laser. Esta escova emite luz vermelha (635nm) no espectro visível que é produzida por um laser diodo que estava dentro da escova de dentes, sendo alimentada por uma bateria. Foi incluído um total de 96 indivíduos no estudo. Os resultados mostraram que, no período experimental de 4 semanas, a utilização da escova de dentes com laser de baixo nível foi eficaz na diminuição da hipersensibilidade dentinária. No entanto, um período mais longo de acompanhamento pode ser necessário para avaliar os efeitos contínuos dessa modalidade de tratamento.

\section{CONCLUSÃO}

Por meio deste trabalho conclui-se que a utilização dos lasers no tratamento da hipersensibilidade dentinária apresenta-se como uma alternativa clínica bastante eficaz. Diversos protocolos e dosagens de aplicação dos lasers, sejam eles de alta ou baixa potência podem ser utilizados, de forma que, ainda existem controvérsias em qual protocolo apresenta-se mais efetivo. Os estudos demonstram resultados satisfatórios na redução da dor em dentes que foram tratados com o laser, ainda que, diferentes mecanismos de atuação possam ser vistos de acordo com a potência do laser que for aplicado.

\section{REFERÊNCIAS}

1. Moraschini V, Costa LS, Santos GO. Effectiveness for dentin hypersensitivity treatment of non-carious cervical lesions: a meta-analysis. Clin Oral Investig. 2018;22(2):617-31.

2. Hashim NT, Gasmalla BG, Sabahelkheir AH, Awooda AM. Effect of the clinical application of the diode laser $(810 \mathrm{~nm})$ in the treatment of dentine hypersensitivity. BMC Res Notes. 2014;7:31.

3. Canali GD, Rached RN, Mazur RF, Souza EM. Effect of erosion/abrasion challenge on the dentin tubule occlusion using different desensitizing agents. Braz Dent J. 2017;28(2):216-24.

4. Davari AR, Ataei E, Assarzadeh H. Dentin hypersensitivity: etiology, diagnosis and treatment; a literature review. J Dent (Shiraz). 2013;14(3):136-45.

5. Matias NMA, Lesão JC, Filho PFM, Silva CHV. Hipersensibilidade dentinária: uma revisão de literatura. Odontol. Clín.-Cient. 2010; 9(3): 205-208.

6. Asnaashari M, Moeini M. Effectiveness of lasers in the treatment of dentin hypersensitivity. J Lasers Med Sci. 2013;4(1):1-7.

7. Silva EL, Januário MVS, Vasconcelos MG, Vasconcelos RG. O uso do laser como alternativa clínica na dentística. Odontol. Clín.-Cient. 2018; 17(2):79-84.

8. Dantas EM, Dantas PMC, Nóbrega FJO, Vasconcelos RG, Aguiar Júnior JN, Queiroz LMG. Tratamento da hipersensibilidade dentinária cervical com laser de baixa potência revisão de literatura. Odontol. Clín-Cient. 2013;12(1):7-11.

9. Costa LM, Cury MS, Oliveria MAHM, Nogueira RD, Geraldo-Martins VR. A utilização da laserterapia para o tratamento da hipersensibilidade dentinária: revisão da literatura. J Health Sci. 2016;18(3):210-16.

10. Belal MH, Yassin A. A comparative evaluation of $\mathrm{CO}_{2}$ and erbium-doped yttrium aluminium garnet laser therapy in the management of dentin hypersensitivity and assessment of mineral 
contente. J Periodontal Implant Sci. 2014;44(5):227-34.

11.Ozlem K, Esad GM, Ayse A, Aslihan U. Efficiency of lasers and a desensitizer agent on dentin hypersensitivity treatment: a clinical study. Niger J Clin Pract. 2018;21(2):225-30.

12. Tabibzadeh Z, Fekrazad R, Esmaeelnejad A, Shadkar MM, Sadrabad ZK, Ghojazadeh M. Effect of combined application of high- and lowintensity lasers on dentin hypersensitivity: A randomized clinical trial. J Dent Res Dent Clin Dent Prospects. 2018;12(1):49-55.

13.Han SY, Kim JS, Kim YS, Kwon HK, Kim BL. Effect of a new combined therapy with nanocarbonate apatite and $\mathrm{co} 2$ laser on dentin hypersensitivity in an in situ model. Photomed Laser Surg. 2014;32(7):394-400.

14.Lopes AO, Eduardo CP, Aranha ACC. Clinical evaluation of low-power laser and a desensitizing agente on dentin hypersensivity. Lasers Med Sci. 2013;30(2):823-29.

15.Lopes AO, de Paula Eduardo C, Aranha ACC. Evaluation of diferente treatment protocols for dentin hypersensivity: na 18-month randomized clinical trial. Lasers Med Sci. 2017;32(5):1023-30.

16.Lopes AO, Aranha. Comparative Evaluation of the Effects of Nd:YAG laser and a desensitizer agent on the treatment of dentin hypersensitivity: a clinical study. Photomed Laser Surg. 2013; 31(3):132-38.

17.Aranha ACC, Eduardo Cde P. Effects of Er:YAG and Er,Cr:YSGG lasers on dentine hypersensitivity. Short-term clinical evaluation. Lasers Med Sci. 2012;27(4):813-18.

18. Yu CH, Chang YC. Clinical efficacy of the Er:YAG laser treatment on hypersensitive dentin. $\mathbf{J}$ Formos Med Assoc. 2014; 113(6):388-91.

19. Ribeiro JPF, Aranha ACC, Real RC, Eduardo CP. Avaliação dos efeitos do laser de Nd:YAG no tratamento da hipersensibilidade dentinária: estudo preliminar. Revista Saúde. 2011;5(4):14-26.

20.Liu Y, Gao J, Gao Y, Xu S, Zhan X, Wu B. In vitro study of dentin hypersensitivity treated by 980-nm diode laser. J Lasers Med Sci. 2013;4(3):111-19.

21. Namour A, Nammour S, Peremans A, Heysselaer D, De Moor RJG. Treatment of dentinal hypersensitivity by means of Nd:YAP laser: a preliminary in vitro study. Sci World J. 2014; 1: 323604.

22.Dantas EM, Amorim FKO, Nóbrega FJO, Dantas PMC, Vasconcelos RG, Queiroz LMG. Clinical efficacy of fluoride varnish and low-level laser radiation in treating dentin hypersensitivity. Braz Dent J. 2016;27(1):79-82.

23.Bal MV, Keskiner I, Sezer U, Açikel C, Saygun I. Comparison of low level laser and argininecalcium carbonate alone or combination in the treatment of dentin hypersensitivity: a randomized split-mouth clinical study. Photomed Laser Surg. 2015;33(4):200-5.

24.Suri I, Singh P, Shakir QJ, Shetty A, Bapat R, Thakur R. A comparative evaluation to assess the efficacy of 5\% sodium fluoride varnish and diode laser and their combined application in the treatment of dentin hypersensitivity. J Indian Soc Periodontol. 2016;20(3):307-14.

25.Femiano F, Femiano R, Lanza A, Lanza M, Perillo L. Effectiveness on oral pain of $808 \mathrm{~nm}$ doide laser used prior to composite restoration for symptomatic non-caroius cervical lesions unresponsive to desensitizing agentes. Lasers Med Sci. 2017;32(1):67-71.

26.Ko Y, Park J, Kim C, Baek SH, Kook YA. Treatment of dentin hypersensitivity with a lowlevel laser-emitting toothbrush: double-blind randomised clinical trial of efficacy and safety. J Oral Rehabil. 2014;41(7):523-31.

\section{CONFLITO DE INTERESSES}

Os autores declaram não haver conflitos de interesse.

\section{AUTOR PARA CORRESPONDENCIA}

\section{Rodrigo Gadelha Vasconcelos}

rodrigogadelhavasconcelos@yahoo.com.br

Submetido em 29/10/2018

Aceito em 12/03/2019 